\title{
Changement climatique : la vérité est-elle au fond du puits? Une analyse des controverses sur les puits de carbone
}

\author{
BRuno LocatelLI, CYRIL LOISEL
}

Le deuxième "sommet de la Terre ", dix ans après celui de Rio qui s'était concrétisé par la signature de conventions internationales, ne suscite pas le même enthousiasme. Pour comprendre les enjeux des négociations qui suivent de tels sommets, NSS revient avec cet article sur les accords de Kyoto, en particulier les controverses que la question des puits de carbone a suscitées. En analysant la confrontation des argumentaires autour d'une mesure précise, à savoir la possibilité pour les États de comptabiliser les puits dans la réduction des émissions à effet de serre, les auteurs montrent combien la scène de la négociation internationale est complexe et traversée par des enjeux où science et politique sont constamment imbriquées.

Depuis l'apparition du concept de puits de carbone dans les négociations sur le changement climatique au début des années 90 , les controverses n'ont pas cessé.

Avant chaque Conférence des Parties, comme celle de La Haye en novembre 2000 et celle de Bonn en juillet 2001, les articles sur le sujet se multiplient. Quelques titres de la presse grand public nous donnent une idée des débats sur les puits de carbone:

"La reforestation ne réduit pas forcément l'effet de serre " (Le Monde, 9 novembre 2000).

"Les puits de carbone de Bush pourraient causer plus d'effet de serre " (La Science au Québec, 3 avril 2001).

"Les puits de carbone, la fausse bonne idée contre la pollution " (La Croix, 25 mai 2001).

Les controverses sur les puits reflètent des incertitudes scientifiques, mais aussi des divergences de points de vue. Les opposants aux puits, que ce soit des scientifiques, des politiques ou des $\mathrm{ONG}^{1}$, refusent qu'ils soient pris en compte au même rang que les autres efforts de lutte contre le changement climatique.

Généralement, deux grandes approches de limitation des gaz à effet de serre sont envisagées : la réduction de la source principale qui est la combustion de carbone fossile (pétrole, gaz et charbon minéral) et le stockage de l'excédent de $\mathrm{CO}_{2}$ dans les puits. Les opposants aux puits voient cette dernière approche comme une échappatoire, la vraie cause à contrecarrer étant les émissions par le carbone fossile. Les promoteurs disent que c'est une solution pour limiter rapidement et à coût raisonnable les concentrations de gaz à effet de serre dans I'atmosphère. Cette option permet d'amoindrir la pression sur le secteur énergétique et de mettre en place une transition plus douce donc plus acceptable politiquement.

Au niveau de la politique internationale, les négociations ont montré un antagonisme fort entre le groupe de "I'ombrelle " (Canada, États-Unis, Japon, Fédération de Russie, Australie, Nouvelle-Zélande, Norvège, Islande et Ukraine) qui cherche à imposer l'inclusion des puits et l'Europe qui est prudente sur la question, voire opposée. Les pays du Sud sont divisés sur ce sujet; parmi eux, les petits pays insulaires rassemblés dans le groupe Aosis (Alliance of Small Island States) y sont opposés.

Les controverses portent sur les puits en tant qu'objets politiques, c'est-à-dire sur l'inclusion des puits dans le Protocole de Kyoto. Mais elles sont alimentées par des incertitudes, voire des controverses scientifiques, sur les puits, en tant qu'objets physiques. Il est intéressant de noter l'abondance des articles scientifiques sur le changement climatique dans les semaines qui précèdent les conférences internationales sur le sujet (un exemple est donné pour la revue New Scientist dans la figure 1).

La distinction entre la définition physique et la définition politique des puits est souvent ignorée ou sous-estimée, ce qui contribue à brouiller le débat. Dans cet article, une première partie rappellera les définitions. Pour appuyer leurs positions, les opposants et défenseurs de l'inclusion des puits avancen de nombreux arguments. L'article présentera d'aborc

Dans ce même numéro, le texte de $M$. Lefèvre apporte un éclairage complémentaire, car la lutte contre le changement climatique n'est pas qu'une affaire d'États et de scientifiques. $\overline{\text { BRUNO LOCATELLII }}$ Économiste de l'environnement, Cirad Forêt-CATIE, Apdo 2, 7170 Turrialba. Costa Rica, bruno.locatelli@cirad.fr

CYRIL LOISEL Chargé de mission Effet de serre, expert forêt et changement climatique, Office national des forêts. 2, avenue de Saint-Mandé, 75570 Paris cedex 12. cyril.loisel@onf.fr

Comme WWF et GreenPeace (Cadman, 2001), Climate Action Network (2000), World Rainforest Movement (Lohmann, 2000) et Friend of the Earth (2000). Plusieurs ONG participent à la rédaction de la lettre d'information Eco, disponible sur http://www.climatenetwork.org/eco/ 


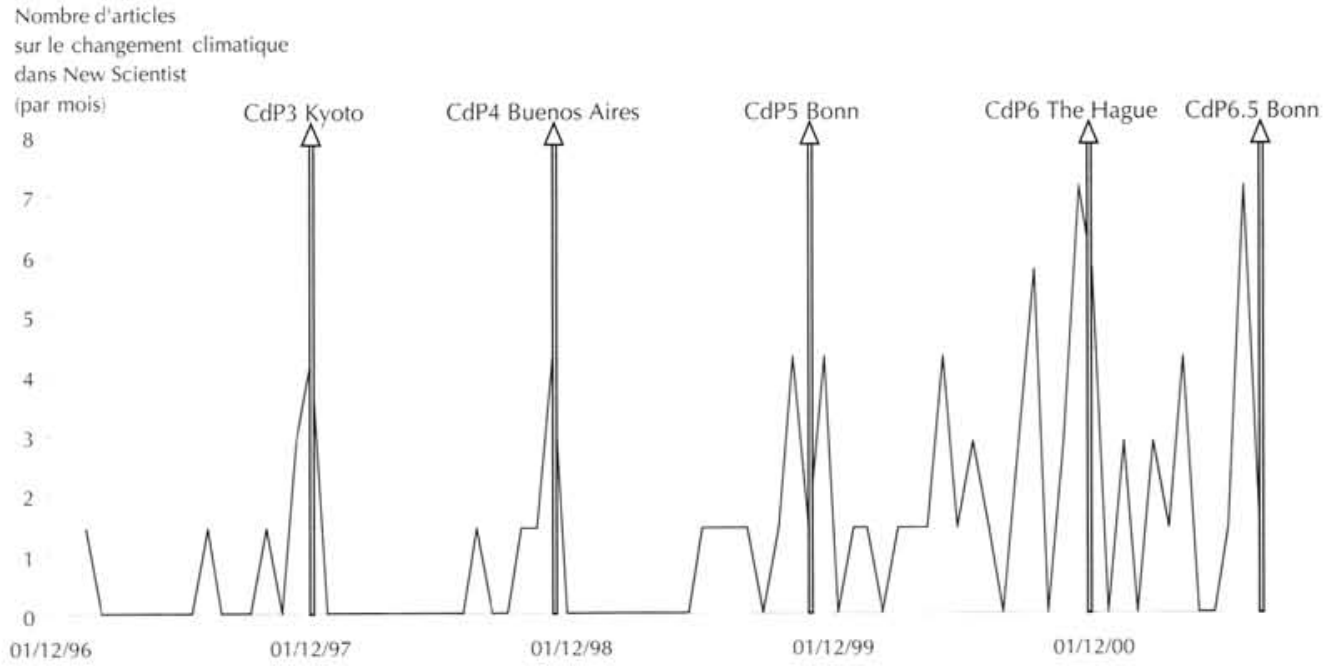

Figure 1. Parution d'articles sur le changement climatique avant les conférences des parties.

Abstract - Climatic change: is the truth at the bottom of the carbon sink?

A main part of the international negotiations on the Kyoto Protocol implementation is focusing on carbon sinks. The term of "carbon sinks" describes the activities related to land use, land-use change and forestry. In general, two main approaches for mitigating climate change are distinguished: the reduction of greenhouse gases emissions caused by fossil fuel consumption and cement production, the sequestration of carbon in sinks. The debates on the inclusion of carbon sinks must be replaced in the context of the discussions on supplementary in the Kyoto Protocol. Since the appearance of the carbon sink concept during the negotiations on climate change in the early 90 s, the controversies have not ceased. These controversies reflect the scientific uncertainties and the diversity of opinion. Both promoters and opponent use scientific results, but sometimes with bias. Some debates are mixed up by the various definitions of sinks as physical objects and sinks as political objects. Some controversies deal with the risk of including sinks in the means for mitigating climate change. The risk is to alleviate the environmental integrity of the Kyoto Protocol. According to the opponents, the scientific basis of carbon sinks is uncertain. Many arguments are given: boreal reforestation may worsen the global change, the current sinks may become large sources in some years because of the effects of atmospheric and climatic changes, the permanence of carbon sequestration in sinks can not be guaranteed, the additionality of carbon sequestration by projects can not

${ }^{2}$ La question de la supplémentarité peut se formuler de la façon suivante: un pays avec des engagements de réductions d'émissions peut-il recourir sans limitation à des instruments de flexibilité (par exemple l'achat de crédits carbone) pour remplir son contrat? Ou doit-il obligatoirement réaliser une partie de ses engagements par des réductions d'émissions dans son secteur énergétique national ? be evaluated, the carbon sink projects may have negative impact on greenhouse gas emissions outside their boundaries, the measurement of carbon sequestration is not efficient or precise. Some other controversies are related to the consequences of carbon sinks on environment and sustainable development, especially on biodiversity. The opponents to carbon sinks warn off the risk of transformation of natural ecosystems into mancreated plantations. Furthermore, the implementation of carbon sinks in the developing countries raises some ethical questions. 2002 Éditions scientifiques et médicales Elsevier SAS international negotiations / CDM (Clean Development Mechanism) climate change / Kyoto Protocol / sink / carbon / forest / les arguments relatifs à l'intégrité environnementale du Protocole de Kyoto. Ensuite seront détaillés ceux relatifs aux impacts des activités " puits "sur le développement durable et la biodiversité. Enfin, les puits seront replacés dans le contexte plus général de la supplémentarité ${ }^{2}$ et leurs impacts sur la dilution des efforts seront abordés.

Les analyses qui suivent sont conduites en considérant les décisions prises le 24 juillet 2001 à la deuxième partie de la Sixième Conférence des Parties de Bonn (CdP, 2001).

\section{Qu'est-ce qu'un puits de carbone?}

\section{Le puits, objet physique}

Par opposition à une source, un puits de carbone se définit comme un réservoir de carbone (un océan ou un écosystème par exemple) qui, pendant une période donnée, absorbe globalement plus de carbone qu'il n'en rejette.

Les deux grandeurs physiques associées sont les stocks, qui caractérisent les réservoirs et les flux de carbone. Les puits sont définis par des stocks croissants ou des flux positifs de l'atmosphère vers la biosphère.

À propos des écosystèmes continentaux, le terme de puits de carbone peut caractériser un objet physique à des échelles différentes, depuis la parcelle forestière jusqu'à la biosphère globale. Par exemple, à l'échelle mondiale, la biosphère terrestre constitue actuellement un puits de carbone qui absorbe chaque année environ 2,3 milliards de tonnes de carbone (figure 2).

Cette valeur est estimée par différence entre les émissions de carbone fossile $(6,3 \mathrm{Gt} / \mathrm{an})$ et l'accumulation dans I'atmosphère (3,3 Gt/an) et dans les océans (2,3 Gt/an), ce qui représente un puits net de 


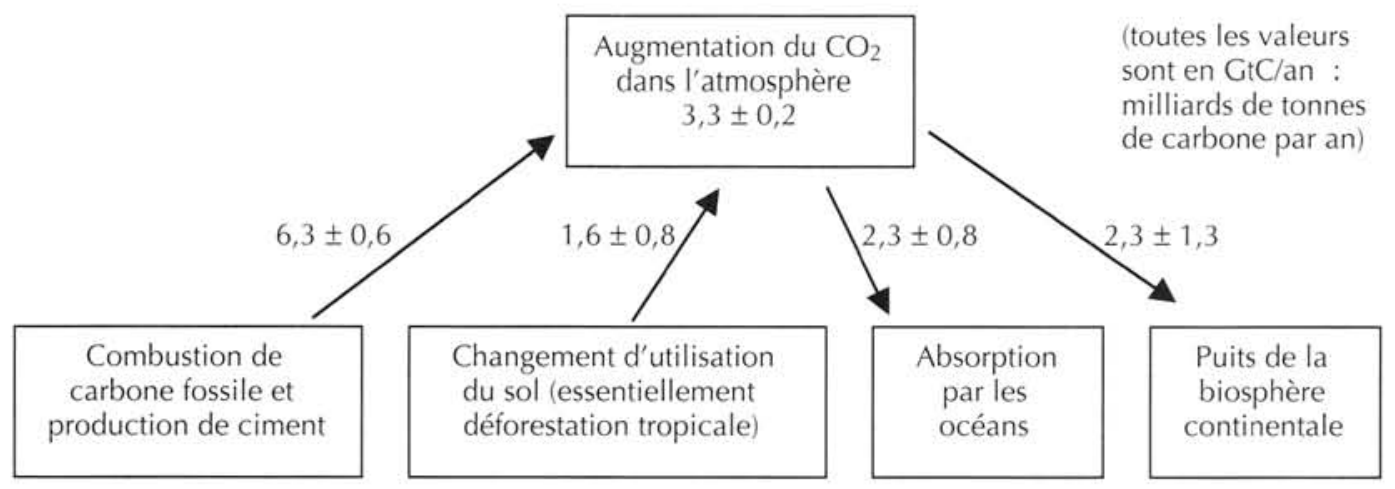

Figure 2. Flux de carbone entre l'atmosphère, les océans et la biosphère continentale pour la période 1989-1998 (d'après Watson et al., 2000).

0,7 Gt/an (Watson et al., 2000). En tenant compte des émissions liées à la déforestation tropicale (estimée à $1,6 \mathrm{Gt} / \mathrm{an})$, on estime que la biosphère continentale absorbe par ailleurs environ 2,3 Gt/an.

Cette absorption se fait en partie par la reforestation des zones tempérées (environ $0,7 \mathrm{Gt}$ /an d'après Dixon et al., 1994). Le reste pourrait être lié à une stimulation de la croissance actuelle des forêts. Cette stimulation s'expliquerait par l'augmentation de la photosynthèse liée à des teneurs accrues en $\mathrm{CO}_{2}$, l'augmentation de la durée de la saison de croissance liée au réchauffement et l'augmentation des dépôts azotés fertilisants liés à la pollution atmosphérique.

\section{Puits naturels et puits gérés}

Deux catégories de puits peuvent être distinguées en fonction de l'influence anthropique. Par opposition aux puits gérés, un puits naturel est un écosystème qui augmente son stock de carbone sans intervention directe de l'homme (néanmoins avec des influences indirectes des activités humaines, comme par exemple la fertilisation par l'augmentation du $\mathrm{CO}_{2}$ atmosphérique).

Sans intervention humaine, une forêt ou une plantation absorbe du carbone pendant sa croissance : c'est un puits (figure 2). Ensuite, lorsqu'elle arrive à maturité, on considère généralement qu'elle est à peu près à l'équilibre. Elle prélève à peu près autant de carbone qu'elle en émet dans l'atmosphère : le puits est dit faible ou inactif (figure 3).

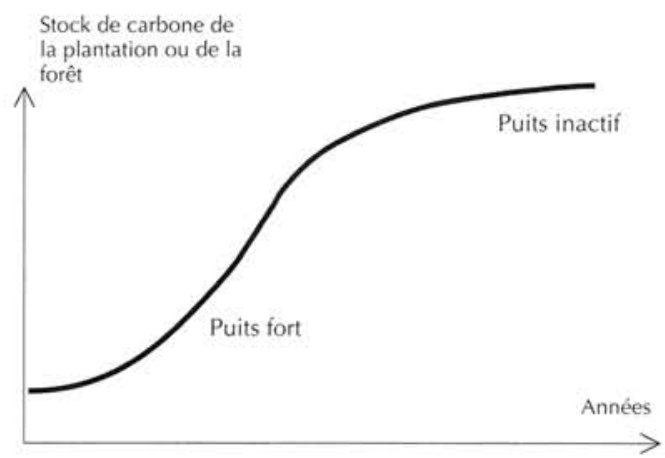

Figure 3. Modèle simplifié des stocks et flux de carbone dans une forêt plantée.
En réalité, une forêt mature n'est pas exactement neutre en terme de flux. Elle peut être soit un puits, soit une source, mais faible en comparaison avec un puits actif. Selon le modèle de Cox-Hadley décrit plus loin, le changement global transformera cet ancien puits en source.

Dans le cas d'un puits géré, la forêt ou la plantation n'a pas pour vocation à rester dans un état mature : elle doit être exploitée et renouvelée. Après une exploitation durable, l'écosystème forestier reconstitue son stock en redevenant un puits actif. Le bilan de l'exploitation en terme de carbone dépendra du devenir des produits. Si tout le bois est abandonné ou non valorisé, le bilan sera négatif. Cependant, si le bois est utilisé comme matériau ou comme énergie, le bilan peut être positif, en particulier si la valorisation énergétique évite la consommation de carbone fossile (substitution). Ce sont les pratiques de gestion des puits et de valorisation des produits qui permettent d'optimiser les bénéfices des puits de carbone pour la réduction du changement climatique.

\section{Quels puits dans le Protocole de Kyoto?}

Lors des négociations internationales sur le changement climatique, les questions relatives à la prise en compte des émissions et absorptions de carbone liées à l'utilisation des sols et à la sylviculture forment un sujet de négociation en soi, désigné par l'acronyme

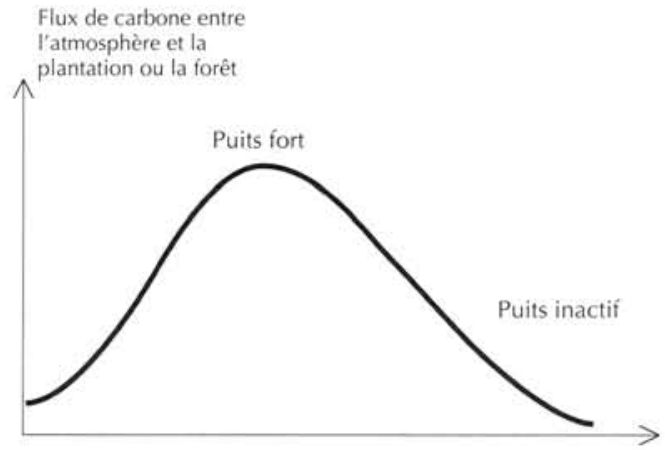


LULUCF (Land Use, Land-Use Change and Forestry), ou plus communément par "les puits".

Dans le Protocole de Kyoto, les puits concernent deux échelles spatiales différentes. La première est macroscopique : il s'agit de la biosphère à l'échelle nationale, qui interviendra dans la comptabilité nationale des pays ayant pris des engagements de réduction (les pays industrialisés ou "pays de I'Annexe I»). Ce ne sont pas les flux de carbone de l'objet physique dans sa totalité qui seront pris en compte, mais seulement les flux causés par certaines activités humaines. D'après l'article 3.3 du Protocole de Kyoto, ces activités sont le boisement, le reboisement et le déboisement. D'après l'article 3.4, d'autres activités peuvent être incluses, comme certaines activités de gestion forestière. Le stockage de carbone compté sous cet article doit résulter d'activités anthropiques ayant eu lieu depuis 1990. Outre l'absence de précision sur le type d'activités concernées, un autre problème réside dans le caractère anthropique. La séparation entre la part du puits due aux activités humaines récentes et celle due à la nature n'est pas toujours scientifiquement réalisable. L'existence de cet article peut s'expliquer par le fait que certains pays avait donné leur accord sur l'engagement de Kyoto à condition qu'une part significative de puits soit prise en compte.

La seconde échelle est locale, il s'agit de projets puits, comme des plantations ou des forêts gérées. Ces projets interviendront dans le Protocole par le biais de deux mécanismes: le Mécanisme pour un développement propre (MDP) pour des projets dans les pays du Sud et la Mise en œuvre conjointe (MOC) pour des projets dans les pays de l'Annexe I. C'est seulement par le biais du MDP que les puits tropicaux seront intégrés à la lutte contre le changement climatique.

Les deux échelles spatiales se réfèrent à deux approches de la comptabilité carbone : une approche consolidée dans le premier cas et une approche par projet dans le second.

\section{L'importance quantitative des puits}

Compte tenu des engagements pris par les pays du Nord et de l'inclusion des puits décidée par le Protocole de Kyoto et les Conférences des Parties, nous pouvons nous interroger à propos de l'importance quantitative des puits par rapport aux efforts nécessaires de réduction d'émissions.

D'après Yamagata et Alexandrov (2001), I'inclusion de toutes les activités " boisement, reboisement et déboisement "des pays de l'Annexe I représenterait une absorption de $200 \mathrm{MtC} / \mathrm{an}$. Au contraire, d'après Watson et al. (2000) l'inclusion de ces activités représenterait une émission, ce qui serait alors encore plus contraignant pour ces pays. II n'est pas rare de rencontrer de telles variations entre les résultats de deux études portant sur des projections d'émissions. Elles peuvent souvent s'expliquer par des différences de définitions des activités de boisement, reboisement et déboisement. Dans le cas présent, les différences s'expliquent principalement par la méthode de prévision des évolutions à venir des surfaces boisées. La première étude repose sur un modèle intégré de l'évolution de l'occupation du sol alors que la seconde suppose que les activités " boisement, reboisement et déboisement " de 1990 vont continuer au même niveau pendant 20 ans.

Nous proposons une évaluation de l'importance quantitative des puits dans leur ensemble, sans se limiter aux activités de boisement, reboisement et déboisement des pays de l'Annexe I. Cette évaluation se base sur des données utilisées lors des négociations de juillet 2001 à Bonn et extraites des communications nationales des pays (tableau I).

Dans le tableau I, sont rapportés les crédits ou débits que procureraient les activités de boisement, reboisement et déboisement (colonne 2) et la gestion forestière (colonne 3). Dans la colonne 4, se trouvent les autres activités : restauration du couvert végétal, gestion des pâturages et gestion des terres cultivées. Toutes ces valeurs concernent les puits des pays de I'Annexe I. Deux totaux ont été calculés, avec et sans les États-Unis, compte tenu de leur prise de position vis-à-vis du Protocole de Kyoto.

Ces données de base ont permis de calculer la contribution des puits à remplir les engagements du Protocole de Kyoto (tableau II). Ces valeurs ont été calculées en appliquant les limites fixées par l'accord de Bonn.

Dans le tableau II, la deuxième colonne concerne l'article 3.3 (boisement, reboisement et déboisement), elle reprend les valeurs de la deuxième colonne du tableau I. L'article 3.4 est plus complexe : les pays peuvent y recourir pour compenser d'éventuels débits de l'article 3.3, dans la limite de 8,2 MtC/an (colonne 3). Au-delà, ils peuvent y recourir dans une certaine limite fixée par pays dans l'appendice $Z$ de l'accord de Bonn (colonne 4). Cette liste de quotas négociés entre les Parties donne un avantage à certains pays : le Canada, le Japon, la Russie et les États-Unis (au cas où ils entreraient de nouveau dans le Protocole) peuvent créditer entre 12 et $28 \mathrm{MtC} / \mathrm{an}$ supplémentaires pour la gestion forestière alors que chaque pays de I'Union Européenne est limité à $0,35 \mathrm{MtC} / \mathrm{an}$ en moyenne.

La cinquième colonne (autres activités additionnelles) reprend les valeurs du tableau I (autres activités). Enfin, la sixième colonne représente la limite du recours du MDP forestier : chaque pays de I'Annexe I peut y recourir dans la limite de $1 \%$ de ses émissions de 1990, comme il fut décidé à Bonn.

Le total pour l'Annexe I est de 159 millions de tonnes de carbone par an (et de 104 millions de tonnes sans les États-Unis). Ce recours potentiel aux puits est à comparer aux efforts nécessaires de réduction d'émission. Le Protocole de Kyoto fixe comme objectif une réduction de $5 \%$ des émissions pendant la période 2008-2012 par rapport aux émissions de 1990. Cela ne signifie pas que les efforts de réduction sont équivalents à ces $5 \%$. En effet, en l'absence d'efforts spécifiques, les émissions auraient continué à croître selon un scénario qu'il est convenu d'appeler "scénario de référence " (figure 4).

Treize scénarios de référence, basés sur différents modèles économiques, ont été passés en revue par 
Tableau I. Données de base par pays de l'Annexe I (toutes les valeurs sont en MtClan ou millions de tonnes de carbone par an).

\begin{tabular}{|c|c|c|c|c|}
\hline Pays & $\begin{array}{l}\text { Boisement, } \\
\text { reboisement et } \\
\text { déboisement }\end{array}$ & $\begin{array}{l}\text { Gestion } \\
\text { forestière }\end{array}$ & Autres activités & Total \\
\hline $\begin{array}{l}\text { Autriche } \\
\text { Belgique } \\
\text { Danemark } \\
\text { Finlande } \\
\text { France } \\
\text { Allemagne } \\
\text { Grèce } \\
\text { Irlande } \\
\text { Italie } \\
\text { Luxembourg } \\
\text { Pays-Bas } \\
\text { Portugal } \\
\text { Espagne } \\
\text { Suède } \\
\text { Royaume-Uni }\end{array}$ & $\begin{array}{r}-0,2 \\
0,1 \\
-1,1 \\
-1,7 \\
-0,2 \\
\\
0,9 \\
0,5 \\
0,0\end{array}$ & $\begin{array}{l}5,2 \\
0,2 \\
0,3 \\
2,2 \\
7,6 \\
8,5 \\
0,6 \\
0,4 \\
1,2 \\
0,1 \\
0,0 \\
1,5 \\
4,5 \\
4,6 \\
2,5\end{array}$ & 0,3 & $\begin{array}{l}5,0 \\
0,2 \\
0,4 \\
1,1 \\
5,9 \\
8,2 \\
0,6 \\
1,3 \\
1,7 \\
0,1 \\
0,0 \\
1,5 \\
4,5 \\
4,5 \\
3,3\end{array}$ \\
\hline Total Union européenne & $-1,3$ & 39,1 & 0,3 & 38,1 \\
\hline $\begin{array}{l}\text { Australie } \\
\text { Biélorussie } \\
\text { Bulgarie } \\
\text { Canada } \\
\text { Croatie } \\
\text { République Tchèque } \\
\text { Estonie } \\
\text { Hongrie } \\
\text { Islande } \\
\text { Japon } \\
\text { Lettonie } \\
\text { Liechtenstein } \\
\text { Lituanie } \\
\text { Monaco } \\
\text { Nouvelle-Zélande } \\
\text { Norvège } \\
\text { Pologne } \\
\text { Roumanie } \\
\text { Fédération de Russie } \\
\text { Slovaquie } \\
\text { Slovénie } \\
\text { Suisse } \\
\text { Ukraine } \\
\text { États-Unis }\end{array}$ & $\begin{array}{r}7,7 \\
0,0 \\
-8,2 \\
0,0 \\
-7,2\end{array}$ & $\begin{array}{r}0,0 \\
2,7 \\
9,1 \\
2,1 \\
1,2 \\
1,9 \\
0,0 \\
11,4 \\
2,5 \\
\\
2,0 \\
\\
4,8 \\
0,0 \\
5,5 \\
7,4 \\
117,5 \\
3,4 \\
1,9 \\
0,3 \\
0,1 \\
288,0\end{array}$ & 0,1 & $\begin{array}{r}2,2 \\
0,0 \\
2,7 \\
9,3 \\
0,0 \\
2,1 \\
1,2 \\
1,9 \\
0,1 \\
10,6 \\
2,5 \\
0,0 \\
2,0 \\
0,0 \\
12,5 \\
0,1 \\
5,5 \\
7,4 \\
109,3 \\
3,4 \\
1,9 \\
0,3 \\
0,1 \\
291,2\end{array}$ \\
\hline Total Annexe I & $-14,0$ & & 17,5 & 504,2 \\
\hline Total Annexe I sans les États-Unis & $-6,8$ & 212,7 & 7,1 & 213,0 \\
\hline
\end{tabular}

le Groupe intergouvernemental d'experts sur le climat (Nakicenovi et al., 2000). Suivant les scénarios, les émissions de 2010 auraient représenté entre $98 \%$ et $114,6 \%$ des émissions de 1990 . La moyenne est de $106 \%$, soit un effort de réduction équivalent à $11 \%$ des émissions de 1990.

Pour simplifier, nous considérons que ce pourcentage est le même selon que les États-Unis sont pris en compte ou non. Comme les émissions de 1990 sont de l'ordre de $4900 \mathrm{MtClan}$ pour toute l'Annexe I (et $3300 \mathrm{MtC} / a n$ sans les États-Unis), les efforts sont donc de $540 \mathrm{MtC} / \mathrm{an}$ (et $360 \mathrm{MtC} / \mathrm{an}$ pour I'Annexe I sans les États-Unis). Dans le scénario moyen, les puits représenteraient environ $30 \%$ des efforts de réduction d'émission (figure 5). Dans le scénario d'émission le plus bas, les puits couvriraient la totalité des efforts, alors que dans le scénario élevé, ils représenteraient environ $16 \%$.

Les opposants à l'inclusion des puits considèrent probablement que le recours aux puits jusqu'à $30 \%$ des efforts est une échappatoire face aux véritables mesures à prendre dans les secteurs de l'énergie, de l'industrie ou des transports. Au contraire, ce chiffre peut être considéré comme faible par les parties 
Tableau II. Limites du recours aux puits par pays de l'Annexe I (toutes les valeurs sont en M:Clan).

\begin{tabular}{|c|c|c|c|c|c|c|}
\hline Pays & $\begin{array}{l}\text { Article } 3.3 \text { : } \\
\text { boisement, } \\
\text { reboisement et } \\
\text { déboisement }\end{array}$ & $\begin{array}{c}\text { Article } 3.4: \\
\text { gestion } \\
\text { forestière } \\
\text { (compensation } \\
\text { des débits } \\
\text { de l'article } 3.3 \text { ) }\end{array}$ & $\begin{array}{c}\text { Article } 3.4 \text { et } 6: \\
\text { gestion } \\
\text { forestière } \\
\text { (au-delà } \\
\text { compensation) }\end{array}$ & $\begin{array}{c}\text { Article } 3.4: \\
\text { autres } \\
\text { activités } \\
\text { additionnelles }\end{array}$ & $\begin{array}{l}\text { Article } 12: \\
\text { reboisements } \\
\text { dans le MDP } \\
\text { (à supposer } \\
\text { que chaque } \\
\text { pays remplisse } \\
\text { son quota) }\end{array}$ & $\begin{array}{c}\text { Total } \\
\text { des puits }\end{array}$ \\
\hline $\begin{array}{l}\text { Autriche } \\
\text { Belgique } \\
\text { Danemark } \\
\text { Finlande } \\
\text { France } \\
\text { Allemagne } \\
\text { Grèce } \\
\text { Irlande } \\
\text { Italie } \\
\text { Luxembourg } \\
\text { Pays-Bas } \\
\text { Portugal } \\
\text { Espagne } \\
\text { Suède } \\
\text { Royaume-Uni }\end{array}$ & $\begin{array}{r}-0,2 \\
0,1 \\
-1,1 \\
-1,7 \\
-0,2 \\
-0,9 \\
0,5 \\
0,0 \\
\\
-0,1 \\
0,6\end{array}$ & $\begin{array}{r}-0,2 \\
-1,1 \\
-1,7 \\
0,2 \\
\\
\\
\\
\\
\\
-0,1 \\
0,6\end{array}$ & $\begin{array}{l}0,6 \\
0,0 \\
0,1 \\
0,2 \\
0,9 \\
1,2 \\
0,1 \\
0,1 \\
0,2 \\
0,0 \\
0,0 \\
0,2 \\
0,7 \\
0,6 \\
0,4\end{array}$ & 0,3 & $\begin{array}{l}0,2 \\
0,4 \\
0,2 \\
0,2 \\
1,5 \\
3,3 \\
0,3 \\
0,1 \\
1,4 \\
0,0 \\
0,6 \\
0,2 \\
0,8 \\
0,2 \\
2,1\end{array}$ & $\begin{array}{l}0,8 \\
0,4 \\
0,3 \\
0,4 \\
2,4 \\
4,5 \\
0,4 \\
1,1 \\
2,1 \\
0,0 \\
0,6 \\
0,4 \\
1,5 \\
0,8 \\
3,3\end{array}$ \\
\hline $\begin{array}{l}\text { Total Union } \\
\text { européenne }\end{array}$ & $-1,3$ & 3,3 & 5,2 & 0,3 & 11,6 & 19,1 \\
\hline $\begin{array}{l}\text { Australie } \\
\text { Biélorussie } \\
\text { Bulgarie } \\
\text { Canada } \\
\text { Croatie } \\
\text { République Tchèque } \\
\text { Estonie } \\
\text { Hongrie } \\
\text { Islande } \\
\text { Japon } \\
\text { Lettonie } \\
\text { Liechtenstein } \\
\text { Lituanie } \\
\text { Monaco } \\
\text { Nouvelle-Zélande } \\
\text { Norvège } \\
\text { Pologne } \\
\text { Roumanie } \\
\text { Fédération de } \\
\text { Russie } \\
\text { Slovaquie } \\
\text { Slovénie } \\
\text { Suisse } \\
\text { Ukraine } \\
\text { États-Unis }\end{array}$ & $\begin{array}{r}-8,2 \\
0,0 \\
-7,2\end{array}$ & $-4,4$ & $\begin{array}{r}0,0 \\
0,4 \\
12, \\
0,3 \\
0,1 \\
0,3 \\
0,0 \\
13,0 \\
0,3 \\
0,0 \\
0,3 \\
0,0 \\
0,2 \\
0,4 \\
0,8 \\
1,1 \\
\\
17,6 \\
0,5 \\
0,4 \\
0,5 \\
1,1 \\
28,0\end{array}$ & 0,1 & $\begin{array}{l}1,3 \\
0,0 \\
0,3 \\
1,6 \\
0,0 \\
0,5 \\
0,1 \\
0,2 \\
0,0 \\
3,3 \\
0,1 \\
0,0 \\
0,1 \\
0,0 \\
0,2 \\
0,1 \\
1,3 \\
0,6\end{array}$ & $\begin{array}{r}3,5 \\
0,0 \\
0,7 \\
18,2 \\
0,0 \\
0,8 \\
0,2 \\
0,5 \\
0,1 \\
16,3 \\
0,4 \\
0,0 \\
0,4 \\
0,0 \\
8,1 \\
0,6 \\
2,1 \\
1,7\end{array}$ \\
\hline Total Annexe I & $-14,0$ & 23,8 & 82,5 & 17,5 & 49,2 & 159,1 \\
\hline $\begin{array}{l}\text { Total Annexe I } \\
\text { sans les États-Unis }\end{array}$ & $-6,8$ & 16,7 & 54,5 & 7,1 & 32,7 & 104,1 \\
\hline
\end{tabular}

adverses, notamment les gouvernements qui ont signé le Protocole de Kyoto dans l'idée que les puits seraient pris en compte de manière relativement large.

Entre les pays de l'Annexe I, I'importance qualitative du recours est très élevée pour la Nouvelle-
Zélande et le Canada qui pourraient atteindre la totalité de leurs engagements. Au contraire, pour I'Union européenne, le recours aux puits ne représenterait qu'environ $12 \%$ des efforts nécessaires de réduction. 


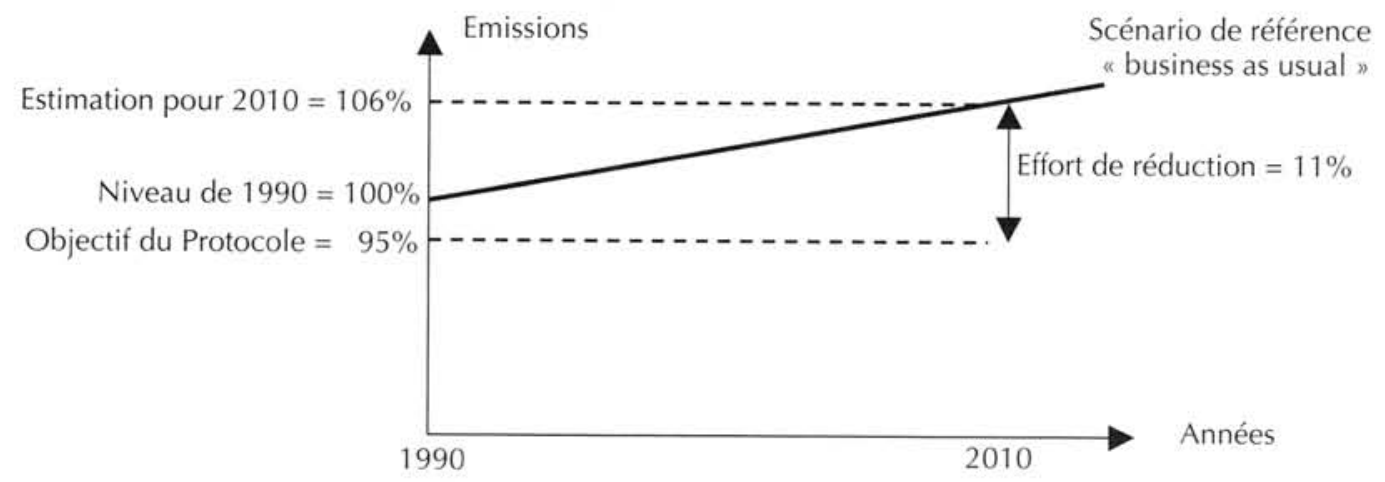

Figure 4. Évolution des émissions et efforts de réduction.

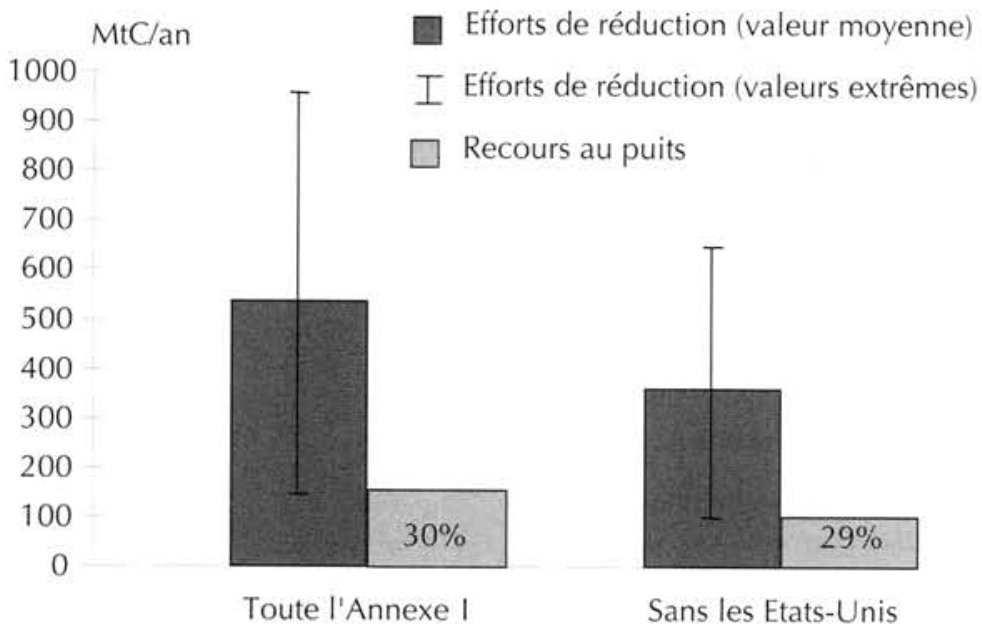

Figure 5. L'importance des puits dans les efforts de réduction.

\section{Les débats sur les puits}

L'inclusion des puits dans la lutte contre le changement climatique fait l'objet de nombreuses critiques, autour de trois thèmes principaux :

- Quelle est la contribution réelle de la séquestration dans le contrôle du changement climatique, en comparaison avec les émissions évitées?

- Quel est l'impact des puits de carbone sur d'autres aspects du développement durable?

- Quel est l'impact des puits de carbone sur la " dilution des efforts de réduction des émissions "?

Ces trois thèmes sont développés dans les parties suivantes.

\section{La contribution des puits au contrôle du changement climatique}

L'objectif ultime de la Convention sur les changements climatiques est la stabilisation de la concentration des gaz à effet de serre dans l'atmosphère à un niveau non dangereux. Le Protocole de Kyoto fixe des engagements de limitation ou de réduction des émissions nettes pour les pays de l'Annexe I. Les puits peuvent mettre en danger l'intégrité environnementale du protocole s'ils permettent davantage d'émissions de carbone fossile sans les compenser réellement par ailleurs.

\section{Les controverses scientifiques sur les puits de carbone}

Certains opposants mettent en évidence les incertitudes scientifiques sur les puits. Ils citent des études sur l'albédo ${ }^{3}$ qui suggèrent que la reforestation pourrait causer davantage de réchauffement dans certaines conditions. Ils avancent également qu'avec l'effet du changement climatique, les puits actuels vont se transformer en " sources ". D'après les opposants, créer des puits revient à créer des bombes à retardement.

\section{L'albédo}

À propos de l'albédo, l'article le plus souvent cité est celui de Betts (2000) paru dans Nature quatre jours 
avant le début de la conférence de La Haye. Dans les zones boréales, une forêt présente un coefficient d'albédo plus faible qu'un terrain nu (souvent recouvert de neige), ce qui contribue à réchauffer le climat, indépendamment du cycle du carbone. Betts calcule que l'effet de l'albédo l'emporte sur l'effet de la séquestration de carbone : planter des arbres en zone boréale ne réduirait pas le réchauffement de la planète. Si ces résultats étaient confirmés par d'autres études, il ne faudrait pas oublier qu'ils ne portent que sur les zones septentrionales et ne peuvent pas être généralisés à la reforestation sur toute la planète.

\section{Le modèle de Cox-Hadley}

Le second point concerne l'effet du changement global sur la biosphère. Les opposants à l'inclusion des puits ont utilisé le modèle de Cox-Hadley pour décrire les puits comme des bombes à retardement. Les puits créés aujourd'hui deviendraient de vastes sources dans quelques décennies.

Ce modèle développé par le Hadley Centre for Climate Prediction and Research intègre les rétroactions entre la biosphère et le climat. En effet, les termes du bilan carbone de la biosphère (absorption par photosynthèse et émission par respiration et décomposition) sont sensibles à l'augmentation du taux de $\mathrm{CO}_{2}$ et aux changements de température.

Quatre jours avant la conférence de La Haye, Nature présente ce modèle (Cox et al., 2000) comme reposant sur des hypothèses d'évolution de la photosynthèse, de la respiration avec les changements globaux.

Ces hypothèses ont été critiquées par ailleurs. Par exemple, dans le modèle, la respiration du sol double quand la température augmente de 10 degrés (Cox, 2001). Dans un article du Monde daté du 10 novembre 2000, Bernard Saugier dit que "cette hypothèse est valide lorsqu'on étudie les variations de respiration saisonnières dans un écosystème donné, mais ne marche plus du tout quand on l'applique pour comparer des climats différents ".

Le modèle montre que la biosphère restera un puits global jusqu'en 2050 avant de devenir une source globale. La prise en compte de cette rétroaction augmente de $40 \%$ l'estimation du réchauffement planétaire en 2100.

Cette vision de la biosphère est critiquable car la gestion des puits par l'homme n'est pas prise en compte. L'article de Cox concerne plutôt des vastes étendues de forêts non gérées en quasi-équilibre. Ces forêts se comportent actuellement comme un puits physique et pourraient devenir une source, faible par unité de surface mais importante en considérant les surfaces en question.

Néanmoins, en l'absence d'action anthropique directe, ces forêts ne sont pas incluses dans le Protocole de Kyoto et ne font donc pas partie des puits au sens politique (comme le disent l'article 3.3: "variations [...] résultant d'activités humaines... » et l'article 3.4 : " activités anthropiques supplémentaires... ")

${ }^{3}$ Le coefficient d'albédo est la fraction d'énergie solaire réfléchie par la surface de la Terre.

\section{Les articles de mai 2001 dans Nature :}

\section{I'effet fertilisant du $\mathrm{CO}_{2}$}

À propos de la fertilisation de la croissance des puits par le $\mathrm{CO}_{2}$, une autre controverse porte sur la réalité de cet effet. Deux études publiées dans Nature le 24 mai 2001 ont eu beaucoup de retentissement. La première (Oren et al., 2001) décrit la croissance d'une forêt de pins de Caroline du Nord plongée dans une atmosphère enrichie en $\mathrm{CO}_{2}$, la concentration étant le double de celle de l'atmosphère avant la Révolution Industrielle. Pendant les deux premières années, la croissance des arbres était plus forte que sur les sites témoins. Ensuite, la croissance s'est ralentie pour atteindre le même niveau que celle des témoins.

Dans le même numéro de Nature, une autre étude (Schlesinger et al., 2001) réalisée sur le même site donne des résultats similaires pour le stockage de carbone dans le sol et la litière. Les études montrent que la stimulation de croissance par le $\mathrm{CO}_{2}$ peut exister seulement si la nutrition minérale du sol n'est pas limitante.

On peut remarquer que des études présentant des conclusions similaires étaient passées inaperçues il y a quelques années. Celles de Nature ont été publiées deux mois avant le début des négociations de Bonn. En 1996, plus de 3000 textes scientifiques avaient déjà été publiés à propos de l'effet de l'augmentation du $\mathrm{CO}_{2}$ sur les plantes (d'après ProClim-OcCC, Forum sur le climat et le changement global, Académie suisse des Sciences naturelles), sans qu'un avis dominant se distingue. Certaines études concluent sur un effet fertilisant notable (Graybill et Idso, 1993). D'autres sont plus nuancées : une atmosphère enrichie en $\mathrm{CO}_{2}$ entraînerait une augmentation ou une diminution de la croissance des arbres en fonction des espèces (Bazzaz et al., 1990) ou en fonction des sols et de la pluviométrie (Conroy et al., 1990).

Les études publiées dans Nature en mai 2001 sont intéressantes car elles portent sur une forêt réelle et non sur des microcosmes comme la plupart des études précédentes. Les résultats montrent que l'excédent de $\mathrm{CO}_{2}$ a un effet dopant sur la croissance des végétaux mais que cet effet, observé actuellement, n'est que transitoire. Par conséquent, les excès de carbone dans l'atmosphère ne seront pas compensés par une absorption de la biosphère qui serait accrue de façon permanente. Par contre, l'étude ne remet pas en cause le fait qu'une plantation ou une forêt en croissance est un puits de carbone qui réduit les quantités de carbone de l'atmosphère. Or, c'est justement ainsi que le message est quelquefois interprété.

Ainsi, selon une dépêche de l'Agence France Presse, deux études scientifiques américaines affirment que : « la plantation de forêt comme moyen de lutte contre le réchauffement climatique pourrait s'avérer un leurre ». Whitfield (2001) dit que " certains décideurs environnementaux espèrent [...] que la plantation de forêt pourrait remplacer une diminution des émissions. Deux études de forêts de pins d'Amérique du Nord montrent que de tels espoirs sont trop optimistes ".

Il est intéressant de noter que l'accent est mis sur l'effet fertilisateur du $\mathrm{CO}_{2}$ et qu'on oublie la simple croissance des plantations ou des forêts. Une plantation qui croît sera toujours un puits de carbone, qu'elle soit ou non stimulée par un taux élevé de $\mathrm{CO}_{2}$. Si on plante 1000 hectares et que le stock dans le sol et la végétation passe en 40 ans de 30 à 150 tonnes de carbone par hectare, alors 3000 tonnes auront été 
prélevées en moyenne chaque année dans l'atmosphère. Avec I'hypothèse d'une fertilisation par le $\mathrm{CO}_{2}$, ce prélèvement aurait peut-être atteint 3200 tonnes par an (qui sait ?). Les articles ont montré qu'il ne fallait pas trop compter sur ces 200 tonnes supplémentaires, mais les opposants aux puits ont aussi effacé les autres 3000 tonnes.

À propos de I'utilisation de ces résultats scientifiques, Brown et al. (2001) font des commentaires similaires : " A recent article in Nature [...] has been widely reported on, and often misinterpreted, by the press [...]. The Oren et al. findings do not undermine the fact that planting new trees where originally there were none (afforestation and reforestation) can remove substantial amount of $\mathrm{CO}_{2}$ from the atmosphere ".

Pour clore le chapitre sur l'effet fertilisateur du $\mathrm{CO}_{2}$, notons que la décision de Bonn a laissé de côté l'absorption causée par les taux de $\mathrm{CO}_{2}$ élevés : " $\mathrm{Ne}$ sont pas comptabilisées les absorptions résultant d'un accroissement des concentrations de dioxyde de carbone par rapport à leur niveau préindustriel " (Alinéa VII.1.h).

\section{Les puits : des solutions limitées et temporaires}

Les opposants aux puits avancent que cette solution est limitée dans l'espace et le temps. D'abord, les surfaces disponibles sur la terre sont restreintes et ne permettront pas d'absorber les émissions par consommation de carbone fossile. Ensuite, le stockage dans les puits est temporaire. La permanence d'un puits peut être mise en péril par de nombreux facteurs physiques (feux, ravageurs), politiques ou économiques. Enfin, même si le stockage était permanent, les puits de carbone ne feraient que remettre à plus tard le problème de réduction des émissions. Un jour ou l'autre, les puits seraient saturés et n'absorberaient plus de carbone.

En effet, les puits représentent une solution limitée mais qui permet de "gagner du temps ", comme l'explique Bernard Saugier dans l'article du Monde. Du point de vue des politiques nationales, les activités " puits " peuvent être plus rapidement mises en œuvre que des changements de technologies d'énergie ou de transport.

Néanmoins, la solution des puits ne peut pas remplacer une réduction des gaz à effet de serre à la source. Dans cet esprit, la décision de Bonn préconise que le recours aux puits dans les mécanismes soit supplémentaire aux autres mesures prises au plan national (voir plus loin).

Quant à la question de la permanence du stockage, elle peut être prise en compte dans les comptabilités nationales ou les mécanismes du Protocole de Kyoto. Si un puits de carbone est détruit, les émissions correspondantes seront mesurées. Non seulement le puits ne pourra plus être utilisé pour compenser des émissions, mais les quantités créditées antérieurement pour la séquestration de carbone seront débitées, pour refléter le fait que le carbone est retourné dans l'atmosphère.

C'est l'objet d'un point de la décision de Bonn : "L'annulation de toute absorption résultant d'activités liées à I'utilisation des terres, au changement d'affectation des terres et à la foresterie est comptabilisée au moment approprié dans le temps » (alinéa VII.1g). Pour le MDP (mécanisme pour un développement propre), des propositions ont été avancées, comme les Crédits Temporaires : le stockage dans un puits donnerait lieu à l'émission d'un crédit-carbone temporaire, à période de validité limitée. Cette période de validité pourrait être prolongée si la séquestration perdure.

La critique de la saturation des puits a déjà été évoquée précédemment : une exploitation raisonnée et une valorisation des produits peuvent réactiver le puits et éviter des émissions grâce à la substitution énergétique.

\section{La mesure, l'additionnalité et les fuites}

Les opposants aux puits mettent en avant le risque d'impliquer, dans la lutte contre le changement climatique, des puits dont on ne peut évaluer la contribution réelle. Estimer la contribution réelle d'un puits revient à mesurer la quantité de carbone, à évaluer l'additionnalité du stockage et à estimer les effets induits. D'après eux, les scientifiques ne savent pas mesurer le carbone absorbé par un puits, ni estimer combien d'émissions de $\mathrm{CO}_{2}$ sont compensées par un puits.

Actuellement, de nombreuses recherches sont en cours sur les mesures de flux ou de stock de carbone ainsi que sur la dynamique du carbone dans les écosystèmes (voir Watson et al., 20004). II est possible d'évaluer des stockages de carbone, avec différentes méthodes, plus ou moins précises et coûteuses. Certes des incertitudes de mesure existent, mais la règle générale est que les risques de surestimation doivent être minimisés. On pourrait imaginer par exemple de créditer un stockage de carbone à la borne inférieure de l'intervalle de confiance de l'estimation du stock. Les mesures de carbone feront par ailleurs l'objet d'une procédure d'audit systématique avant que les crédits-carbone ne soient générés.

D'autres critiques des puits portent sur l'additionnalité et les fuites des projets puits dans le cadre du MDP. En raison des processus socio-économiques avec lesquels interagit un projet, il est difficile de connaître l'effet véritable d'un projet sur les gaz à effet de serre. Deux questions se posent.

La première concerne l'additionnalité : le projet n'aurait-il pas existé de toutes façons, même sans MDP ? La deuxième concerne les fuites: le projet $n$ 'induit-il pas davantage de déforestation (ou d'émissions de carbone en général) en-dehors du périmètre du projet ? Par exemple, si un projet de plantation exclut des populations d'une terre à vocation agricole, cellesci vont probablement aller déboiser d'autres terres. Le bilan des gaz à effet de serre peut alors être négatif.

Ces critiques portent sur les modalités de mise en œuvre des projets puits ou des projets MDP en général. Il existe un risque réel que des méthodes inadaptées entraînent l'éligibilité de projets qui ne génèrent pas de séquestration nette, réelle et additionnelle, ou qui la surestiment. De nombreuses recherches ont lieu actuellement sur ces sujets (Begg, 2000 ; Chomitz, 1998 ; Goldemberg, 1998 ; Kelly, 1999 ; Vine et al., 1999).

(1)

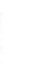

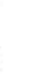




\section{ARTICLE}

Pour évaluer l'additionnalité d'un projet, il faut comparer son stockage de carbone à celui d'un scénario de référence (baseline), qui représente ce qui se serait vraisemblablement passé sans projet. La question est de savoir comment établir des scénarios de référence adaptés et crédibles, sachant qu'ils ne sont que des hypothèses sur ce qui aurait pu se passer sans projet, par nature difficiles à vérifier. Dans le texte de la décision de Bonn, I'alinéa VII.9 rappelle la nécessité de préciser les définitions et les modalités (en particulier sur les questions d'additionnalité et de fuites).

Des enjeux forts reposent sur ces méthodes : si les critères d'éligibilité sont trop stricts, les puits risquent de n'attirer aucun investissement MDP. S'ils sont trop souples, des risques pèsent sur l'intégrité environnementale du Protocole de Kyoto. Néanmoins, ces questions ne sont pas spécifiques aux projets puits, elles se posent également avec la même importance pour tous les types d'activités dans le MDP.

\section{Les effets des puits sur le développement durable}

Les opposants craignent les effets négatifs des puits, outre ceux sur les gaz à effet de serre. Selon eux, les puits présentent des risques pour le développement et l'environnement local. La création de vastes puits de carbone pourrait geler des terres au détriment des populations locales. Certaines ONG dénoncent les problèmes d'éthique ou d'équité Nord/Sud que pose le MDP forestier.

Pour les illustrer, le « World Rainforest Movement " (Lohmann, 2000) utilise l'image de communautés du Sud qui auraient été chassées de leur terre par l'exploitation pétrolière alimentant des consommateurs du Nord, puis chassées à nouveau par des puits de carbone qui compenseraient les émissions de ces mêmes consommateurs. Le terme de " $\mathrm{CO}_{2}$ lonialisme " est apparu récemment ${ }^{5}$.

Selon les opposants, les ressources en eau ou en terres pourraient être dégradées par des plantations à grande échelle. La transformation d'écosystèmes en puits gérés entraînerait une perte de biodiversité. De nombreuses ONG craignent le développement de grandes surfaces de plantations monospécifiques en remplacement des forêts naturelles. Par exemple, le

WWF (WorldWide Fund for Nature) dénonce les effets

${ }^{4}$ Rapport spécial de l'IPCC sur * Land Use, Land Use Change and Forestry". Voir les pages 146 à 158 pour la mesure et

le suivi des activités ARD

(Afforestation, Reforestation and Deforestation), et les pages 319 à 324 pour les projets MDP et MOC

${ }^{5}$ Voir le rapport de Harald Eraker (NorWatch)

à l'adresse

http://www.fivh.no/norwatch/english/Reports/ugan ur la forêt naturelle de certaines plantations à vocation de séquestration de carbone (Cadman, 2001). Cette crainte n'est pas spécifique au MDP et se retrouve dans des critiques de plantations à vocation de production.

Les promoteurs des puits disent au contraire que les conséquences sur le développement et l'environnement seront positives, en utilisant exactement les mêmes arguments mais dans l'autre sens : des projets de reconstitution du couvert boisé auront des effets positifs sur les sols et les régimes hydrologiques. Les activités de reforestation et les écosystèmes reconstitués créeront des emplois, des revenus et des ressources naturelles pour les populations locales.

Qui dit vrai ? Les deux probablement. Les projets forestiers ayant tendance à occuper plus d'espace et à concerner plus de monde que des projets de changement de source d'énergie, on ne peut nier qu'ils auront dans l'ensemble des impacts plus forts sur l'environnement et le développement local, que ces impacts soient positifs ou négatifs.

Ce sont les règles d'éligibilité des projets et les procédures de contrôle qui permettront d'éviter les opérations indésirables et de canaliser les flux d'investissement du MDP vers les projets présentant des cobénéfices pour les populations locales.

L'Accord de Bonn stipule que seuls les projets de boisements seront éligibles au MDP (au moins dans un premier temps) et énonce le principe suivant : * L'exécution d'activités liées à l'utilisation des terres, au changement d'affectation des terres et à la foresterie contribue à la préservation de la diversité biologique et à l'utilisation durable des ressources naturelles " (Alinéa VII.1.e). De plus, le MDP a pour but « d'aider les Parties ne figurant pas à l'Annexe I de parvenir à un développement durable [...] " (Article 12 du Protocole de Kyoto).

Reste à voir comment ces grands principes seront déclinés en règles opérationnelles, ce qui devra être fait à la neuvième Conférence des Parties en 2003. Les débats à venir sont d'une haute importance, d'une part parce que le MDP peut fournir un levier d'action important en faveur du développement et des secteurs forestiers tropicaux, d'autre part parce que le risque de prolifération d'activités indésirables n'est pas négligeable si ce levier est mal pointé. Mais les solutions efficaces et acceptables ne seront pas faciles à trouver, comme en témoignent les difficultés traditionnellement rencontrées lors de l'élaboration de principes forestiers à vocation universelle.

Les promoteurs disent que certains pays attireront les investissements MDP seulement si les puits sont inclus. Il s'agit par exemple des pays les moins avancés, dont les secteurs industriels ou énergétiques ne sont pas suffisamment développés pour attirer des projets d'énergie « propre .

\section{Les puits et la dilution des efforts}

Les opposants craignent que les puits ne diluent les efforts de réduction des émissions. Le risque serait de voir les pays de l'Annexe I remplir leurs engagements essentiellement par la séquestration dans les puits sans modifier leurs politiques nationales en matière d'énergie. Historiquement, la contestation de la séquestration vient du fait que beaucoup de partisans des politiques climatiques placent l'essentiel de l'action nécessaire dans le secteur de l'énergie.

Au cours des négociations internationales, l'enjeu des puits était de faire baisser la tension sur le secteur de l'énergie dans un contexte où il fallait s'assurer la signature de toutes les Parties, hormis les États-Unis. Pour cette raison, le débat sur la dilution par les puits est à replacer dans le contexte plus général de la supplémentarité (Hourcade et Ghersi, 2001).

Au cours des négociations sur le climat, I'Union européenne a insisté pour que les mécanismes de flexibilité (échanges de permis d'émission, mise en 
œuvre conjointe et mécanisme pour un développement propre) soient considérés comme supplémentaires aux actions nationales. Au contraire, le Japon, les États-Unis, le Canada, I'Australie et la NouvelleZélande ont tenu une position opposée sur la supplémentarité. Selon le point de vue de ces pays, limiter le recours aux mécanismes de flexibilité risquait de mettre en péril le Protocole de Kyoto en raison des coûts élevés de mise en œuvre (Hourcade et Le Pesant, 2000).

Si le recours illimité aux mécanismes de flexibilité risque de réduire les efforts nationaux de réductions des émissions, c'est en grande partie parce qu'on peut prédire un prix bas du carbone sur le marché international. II y a à cela de nombreuses raisons qui pourront expliquer ce prix bas, comme la question du " hot air ". Ce terme désigne le quota important de permis que peuvent émettre la Russie et I'Ukraine en raison de la chute de leurs émissions depuis 1990 , I'année de référence. Ajouté aux concessions obtenues par la Russie pour ses propres puits de carbone, le " hot air" laisse présager une offre importante de crédits sur le marché carbone au cours de la première période d'engagement.

De surcroît, le retrait des États-Unis du Protocole de Kyoto, s'il se confirme d'ici l'entrée dans la première période d'engagement en 2008, devrait conduire à une baisse de la demande attendue de crédits carbone. La conjugaison de ces tendances conduirait à un prix potentiel de la tonne de carbone relativement bas.

Au même titre que les mécanismes de flexibilité, les puits de carbone peuvent être vus comme des échappatoires à des efforts sur les réductions d'émissions dans le secteur de l'énergie. L'utilisation des puits pourra avoir un effet déflateur sur les prix de la tonne de carbone et le faible signal-prix n'incitera pas les industriels du Nord à limiter les émissions.

Certaines concessions sur les puits réalisées lors des négociations peuvent être vues comme des formes de dilution des efforts, par rapport aux objectifs fixés par le Protocole de Kyoto. Il s'agit par exemple du quota élevé de crédits carbone liés à la gestion forestière accordé à la Russie. Ainsi, le réseau "Action Climat » dénonce les concessions faites au cours de la septième conférence des parties : "Avec l'inclusion de ces nouvelles concessions, l'objectif de réduction, initialement fixé à $-5,2 \%$ par le Protocole de Kyoto, revient à un objectif de stabilisation des émissions à leur niveau de $1990 x^{6}$.

Au-delà de ces questions sur les concessions nécessaires à l'obtention d'un consensus international, le débat sur les puits et la dilution porte sur les intérêts relatifs des deux stratégies : concentrer les efforts sur la réduction des émissions de carbone fossile ou investir massivement dès aujourd'hui dans la séquestration de carbone.

La séquestration temporaire est économiquement intéressante si les coûts marginaux des dommages du changement climatique sont élevés (Lecocq et Chomitz, 2001). En d'autres termes, si la concentration de gaz à effet de serre dans l'atmosphère est proche d'un "seuil limite " à partir duquel le changement climatique va avoir des conséquences coûteuses, la séquestration (même temporaire) est très souhaitable pour " construire un pont au-dessus de l'abîme ". Dans le cas contraire, la priorité reste aux changements de comportements et de technologies pour infléchir la croissance des émissions de carbone fossile. Cette approche théorique peut difficilement se traduire en orientations politiques : le problème reste de quantifier l'évolution des dommages marginaux du changement climatique.

De nombreuses analyses traitent de l'attrait des puits en fonction des coûts actuels ou futurs de réduction d'émission. La séquestration temporaire apparaît économiquement intéressante si les coûts immédiats de réduction d'émission sont élevés. Dans ce cas, les puits permettront de faire baisser les coûts de réduction des émissions, en attendant que des nouvelles technologies soient développées dans le secteur de l'énergie (Noble et Scholes, 20017).

Les anticipations sur l'évolution des coûts marginaux de réduction et sur le prix du carbone devraient donc jouer un rôle important dans le recours aux puits. En effet, celui-ci sera intéressant si l'opérateur prévoit que le coût présent de séquestration d'une tonne de carbone ajouté au coût futur de réduction d'émission (ou d'achat de permis) est inférieur au coût présent de réduction (ou d'achat d'un permis).

La possibilité d'un retour des États-Unis dans le processus de Kyoto dans le futur (par exemple pour la deuxième période d'engagement) et I'hypothèse d'objectifs plus contraignants (avec notamment la disparition de certaines concessions propres à la première période d'engagement) pourraient conduire les acteurs à anticiper une hausse du prix du carbone. Ceci les inciterait à acquérir le plus tôt possible des crédits carbone plutôt que d'investir dans la séquestration.

\begin{tabular}{|c|c|}
\hline $\begin{array}{l}\text { Résumé - Changement climatique : la vérité est-elle } \\
\text { au fond du puits? } \\
\text { Les activités relatives à l'utilisation des terres et à la } \\
\text { foresterie, regroupées sous le terme de " puits de } \\
\text { carbone », sont un objet de négociation à part entière } \\
\text { pour la mise en ceuvre du Protocole de Kyoto. Les } \\
\text { nombreux débats sur les puits sont à replacer dans le } \\
\text { contexte plus général de la « supplémentarité „ qui traite } \\
\text { de l'association entre deux approches de limitation des } \\
\text { gaz à effet de serre : la réduction des émissions de } \\
\text { carbone fossile par les pays du Nord et le recours aux } \\
\text { mécanismes de flexibilité du Protocole de Kyoto ou aux } \\
\text { puits pour le stockage de l'excédent. De nombreuses } \\
\text { controverses sur l'inclusion des puits dans la lutte contre } \\
\text { le changement climatique accompagnent les } \\
\text { négociations internationales. Promoteurs et détracteurs } \\
\text { utilisent souvent des résultats scientifiques, quelquefois } \\
\text { en les détournant. Les débats sont brouillés par les } \\
\text { multiples définitions des puits, objets physiques et objets } \\
\text { politiques. Certaines controverses portent sur les risques } \\
\text { de l'inclusion des puits pour l'intégrité environnementale } \\
\text { du Protocole. D'autres controverses portent sur les } \\
\text { conséquences des puits sur le développement durable, en } \\
\text { particulier sur la biodiversité. Les puits sont également } \\
\text { critiqués pour leurs impacts sur la dilution des efforts de } \\
\text { réduction d'émission. (O 2002 Éditions scientifiques et } \\
\text { médicales Elsevier SAS } \\
\text { changement climatique / Protocole de Kyoto / puits / } \\
\text { carbone / forêt / négociations internationales / MDP } \\
\text { (mécanisme pour un développement propre) }\end{array}$ & $\begin{array}{l}{ }^{6} \text { Réseau Action Climat, } \\
\text { 2001. Bilan de CoP7 : les } \\
\text { puits de carbone. } \\
\text { Adresse : http://www.rac- } \\
\text { f.org/COP7/Bilan } \\
\text { COP7/p6.htm } \\
7 \text { \& Sinks can be deployed } \\
\text { relatively rapidly at moder- } \\
\text { ate cost and thus could } \\
\text { play a useful bridging role } \\
\text { while new energy techno- } \\
\text { logies are developed. There } \\
\text { is no difference in climato- } \\
\text { logical effect between } \mathrm{CO}_{2} \\
\text { taken up by the land and } \\
\mathrm{CO}_{2} \text { reductions due to } \\
\text { other causes } \text {. }\end{array}$ \\
\hline
\end{tabular}

\section{Résumé - Changement climatique : la vérité est-elle} au fond du puits ? foresterie, regroupees sous le terme de « puits de carbone $»$, sont un objet de négociation à part entière pour la mise en œuvre du Protocole de Kyoto. Les nombreux débats sur les puits sont à replacer dans le contexte plus général de la * supplémentarité " qui traite mécanismes de flexibilité du Protocole de Kyoto ou aux puits pour le stockage de l'excédent, De nombreuses critiqués réduction d'émission. 2002 Éditions scientifiques et médicales Elsevier SAS

changement climatique / Protocole de Kyoto / puits (mécanisme pour un développement propre)

.

(1)

.

.

.

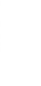


En d'autres termes, si les acteurs du marché du carbone prévoient un faible prix du carbone initial et anticipent un prix nettement plus élevé dans le futur, ils préféreront attendre pour lancer des programmes de développement des puits de carbone.

\section{Conclusion}

Les controverses sur les puits de carbone, ou sur le changement climatique en général, ont accompagné et accompagneront encore les négociations internationales sur le Protocole de Kyoto. Même si elles utilisent parfois des résultats scientifiques de façon un peu détournée, elles sont l'occasion pour opposants ou défenseurs de faire passer des messages, quelquefois de façon très médiatisée.

Cependant, ces controverses ne reflètent pas réellement la nature des débats politiques qui ont lieu lors des Conférences des Parties : les négociations portent sur les règles à appliquer pour rendre le système dans la mesure du possible cohérent et opérationnel et, surtout, acceptable par toutes les Parties.

À Bonn, un accord ne serait pas intervenu si les puits n'avaient pas été intégrés. Au même titre que la supplémentarité, l'inclusion des puits était nécessaire pour aboutir à un accord. Pour cette raison, les questions scientifiques et les controverses sur les puits étaient bien loin des débats politiques. Par exemple, un des résultats des négociations de Bonn porte sur les quotas à attribuer à chaque Partie sur l'article 3.4 (gestion forestière et autres activités). Les valeurs des quotas résultent des positions de force des différentes Parties et non de considérations scientifiques.

Même si le Protocole n'est pas encore appuyé par un nombre suffisant de ratifications pour entrer en vigueur, l'inclusion de certaines activités rurales dans la lutte contre le changement climatique a été confirmée et précisée à Bonn puis à Marrakech. Seront comptabilisées les activités de déboisement, boisement, reboisement, gestion forestière, gestion des sols agricoles et revégétalisation dans les pays de l'Annexe I et les activités de boisement et reboisement dans les pays du Sud par le biais du MDP.

Pour le MDP forestier, nous avons vu l'importance des scénarios de référence et de la prise en compte des fuites négatives ou des impacts sur l'environnement et le développement local. Le texte de la décision de Bonn précise que le Mécanisme pour un développement propre devra démarrer vite et que des modalités et des procédures de mise en œuvre devront être rapidement proposées : des enjeux importants résident dans ces prochaines décisions.

Les discussions relatives à l'intégrité environnementale du Protocole de Kyoto ou aux impacts sur l'environnement et le développement local ne sont donc pas terminées. À peine seront-elles closes en 2003 qu'elles reprendront pour l'élaboration des règles concernant les périodes d'engagement ultérieures, après 2012. Les difficultés de la négociation ressenties à La Haye, pour finalement aboutir à l'accord de Bonn puis celui de Marrakech, ne sont probablement que les prémisses d'autres débats houleux sur le sujet.

\section{Remerciements}

Les auteurs tiennent à remercier Jacques Weber (Cirad), Bernard Saugier (université Paris-Sud) et les relecteurs anonymes pour leurs commentaires. Les opinions exprimées sont celles des auteurs et ne reflètent pas nécessairement celles des relecteurs, du Cirad ou de l'ONF.

\section{BIBLIOGRAPHIE}

Bazzaz, F.A., Coleman, J.S., Morse, S.R., 1990. Growth Responses of Seven Major Co-Occurring Tree Species of the Northeastern United States to Elevated CO2. Can. J. For. Res., 20, 9, 1479-84.

Begg, K. (Ed.), 2000 Initial Evaluation of CDM type projects in Developing Countries. Guildford, UK, Centre for Environmental Strategy, University of Surrey (Disponible à l'adresse : http : //www.surrey.ac.uk/CES/ji/cdmdfid.htm).

Betts, R.A., 2000. Offset of the potential carbon sink from boreal forestation by decreases in surface albedo. Nature, 408, 187-190 (9 November 2000).

Brown, S., Sampson, R.N., Schlamadinger, B., Kinsman, J., 2001. Policy Considerations for Using Forests to Mitigate Carbon Dioxide Emissions. The Scientific World, 1, 241-42 (disponible à l'adresse : http : $/ / 216.25 .253 .201 /$ tsw/iPublish/articles/2001:29.60.pdif).

Cadman, T., 2001. The Kyoto Effect: How the push for Carbon Sinks by Industry and Government has become a Driver for Deforestation. A report for GreenPeace International and WWF. Disponible à l'adresse : http : //www.panda.org/resources/publications/climate/carbonsinks/carbonsinks.html

Chomitz, K. M., 1998. Baselines for Greenhouse Gas Reductions: Problems, Precedents, Solutions. Washington, Carbon Offsets Unit, World Bank (disponible à l'adresse : http ://www.worldbank.org'nipr/work_paper/base14/base14.pdif).

Climate Action Network, 2000. CoP6: CAN position on Land Use, Land Use Change and Forestry. CAN position papers (disponible à l'adresse : http : //www.climatenetwork.org/CAN-COP6-LULUCF.pdt).

Conférence des Parties, 2001. Mise en cuvre du Plan d'Action de Buenos Aires : décision $5 / \mathrm{CP}$. Sixième session de la Conférence des Parties, 2e partie, Bonn, 24 juillet 2001, FCCCCP/20001/L7 idisponible à l'adresse: http : //www.unficcc.int/resource/docs/irench/cop6secpart/cp65107f.pdf).

Conroy J.P., Milham P.J. et al.,1990. Influence of Phosphorus Deficiency on the Growth Response of Four Families of Pinus radiata Seedlings to $\mathrm{CO}_{2}$. Enriched Atmospheres. For. Ecol. Mgmt., 30, 175-88.

Cox P.M., 2001. Description of the TRIFFID dynamic global vegetation model. Hadley Centre, Bracknell, UK. (disponible à l'adresse : http : //www.metoffice.govuk/research/hadleycentre/pubs/HCTN/HCTN_24.pdf).

Cox, P.M., Betts, R.A., Jones, C.D., Spall, S.A., Totterdell, I.J., 2000. Acceleration of global warming due to carbon-cycle feedbacks in a coupled climate model. Nature, 408, 184-87 (9 November 2001).

Dixon, R.K., Brown, S., Houghton, R.A., Solomon, A.M., Trexler, M.C., Wisniewski, I., 1994. Carbon pools and flux of global forest ecosystems. Science, 263, 5144, 185-90.

Friends of the Earth, 2000. The Politics of Climate Change. London, FoE International, Climate Change Briefing (disponible à l'adresse : http : //www.foei.org/Publications/fulladobetext/fccc.pdi).

Goldemberg, I. Ed. 1998. The Clean Development Mechanism: Issues and Options. New York, United Nations Development Programme (disponible à l'adresse : http : //www.undp.org/seed/eap/Publications/1998/1998a.html).

Gravbill, D.A., Idso, S.B., 1993. Detecting the aerial fertilization effect of atmospheric $\mathrm{C} 02$ enrichment in tree-ring chronologies. Global Biogeochemical Cycles, 7, 81-95. 
Hourcade, J.C., Ghersi, F., 2001. The economics of a lost deal. CIRED, Nogent, France, Working Paper (disponible à l'adresse : http : //www.centre-cired.fr/actualite/economiclostdeal.pdf).

Hourcade, I.C., Le Pesant, T., 2000. Negotiating targets, negotiating flex-mex: the economic background of a US-EU controversy. In : Carraro C. (Ed.). Efficiency and equity of climate change policy. Dordrecht : Kluwer Academics Publishers, pp. 63-90.

Kelly, C., 1999. Developing the rules for Determining Baselines and Additionality for the Clean Development Mechanism : Recommendations to the UNFCCC. Washington, Center for Clean Air Policy (disponible à l'adresse: http: //www.cdmcentral.org/docs/baselines/06.pdf).

Lecocq, F., Chomitz, K., 2001. Optimal Use of Carbon Sequestration in a Global Climate Change Strategy: Is there a Wooden Bridge to a Clean Energy Future? Working Papers on, Environment. World Bank, Development Economic Research Group, Infrastructure and Environment, Washington (disponible à l'adresse : http : //econ.worldbank.org/files/2314_wps2635.pdi).

Lohmann, L., 2000. Le marché du carbone : dans le sillage de nouveaux problèmes. World Rainforest Movement, Campaign Material, Montevideo, Uruguay (disponible à l'adresse : http : //www.wm.org.uy/plantations/material/carbonir.rtf).

Nakicenovic, N., Swart, R., 2000. Emissions Scenarios. Special Report of the Intergovernmental Panel on Climate Change. Cambridge University Press, UK (résumé disponible à l'adresse : http : //www.ipcc.chipub/sres-f.pdt).

Noble, I., Scholes, R.J., 2001. Sinks and the Kyoto Protocol. Climate Policy, 1 , 1, 5-25 (résumé disponible à l'adresse : http : //www.elsevier.nl/gejng/10/14/42/33/24/26/abstract.html).

Oren, R., Ellsworth, D.S., Johnsen, K.H., Phillips, N., Ewers, B.E., Maier, C. Schäfer, K.V.R., Mccarthy, H., Hendrey, G., Mcnulty, S.G., Katul, G.C. 2001. Soil fertility limits carbon sequestration by forest ecosystems in a CO2-enriched atmosphere. Nature, 411, 469-72 (2 May 2001).
SBI Subsidiary Body for Implementation, 2000. National communications from Parties included in Annex I to the Convention: Greenhouse gas inventory data from 1990 to 1998. United Nations Framework Convention on Climate Change, note FCCC/SBI/2000/INF.13, The Hague, 11 october 2000 (disponible à l'adresse : http : //www.uníccc.int/resource/docs/2000/sbi/inf13.pdi).

Schlesinger, W.H., Lichter, J., 2001. Limited carbon storage in soil and litter of experimental forest plots under increased atmospheric $\mathrm{CO}_{2}, 2001$ Nature, 411, 466-69 (24 May 2001).

Sturm, M., Racine, C., Tape, K., 2001. Climate change: Increasing shrub abundance in the Arctic. Nature, 411, 546-47 (31 Mav 2001).

Unfccc, 2001. Comparison of Greenhouse Gas Emission Projections. United Nations Framework on Climate Change, Bonn, FCCC/TP/2001/1, 10 july 2001 (disponible à l'adresse. http : //www.unfccc.int/resource/docs/tp/tp0101.pdf).

Vine, E., Sathaye, I., Makundi, W., 1999. Guidelines for the Monitoring, Evaluation, Reporting, Verification, and Certification of Forestry Projects for Climate Change Mitigation. Berkeley, California, Ernest Orlando Lawrence Berkelev National Laboratory. LBNL-41877 idisponible à l'adresse : http : //eetd.lbl.goviea/ies/sunib/climatechange/41543.pdf).

Watson, R.T., Noble, I.A., Bolin, B., Ravindranath, N.H., Verardo, D.J. Dokken, D.J., 2000. Land Use, Land-Use Change and Forestry. Special Report of the Intergovernmental Panel on Climate Change. Cambridge University Press, UK (résumé disponible à l'adresse : http : //www.ipcc.ch/pub/srlulucf-f.pdi).

Whitfield, J., 2001. Sink hopes sink: Forests show little response to increased carbon dioxide. Nature Science Update, 24 May 2001 (disponible à l'adresse : http : //www.nature.com/nsu/010524/010524-14.html).

Yamagata, Y., Alexandrov, G.A., 2001. Would forestation alleviate the burden of emission reduction? An assessment of the future carbon sink from ARD activities. Climate Policy, 1, 1, 27-40 résumé disponible à l'adresse: http : //www.elsevier.nl/gej-ng/10/14/42/33/24/27/abstract.html. 